\title{
El Genoma en los Cordados: Introducción a la Genómica Comparada
}

\author{
The Genome in the Chordates: Introduction to Comparative Genomics
}

"Rodolfo E. Ávila; "María Elena Samar; *"Leticia Díaz-Beltrán \& **Francisco J. Esteban

\begin{abstract}
ÁVILA, R. E.; SAMAR, M. E.; DÍAZ-BELTRÁN, L. \& ESTEBAN, F. J. El genoma en los cordados: introducción a la genómica comparada. Int. J. Morphol., 30(4):1309-1315, 2012.

RESUMEN: El objetivo del presente trabajo es describir brevemente conceptos de la genómica de los cordados así como de la genómica comparada y sus aspectos éticos. El genoma de los cordados cambió ligeramente dando lugar al genoma de los vertebrados, entre ellos el de los mamíferos, entre los que se incluye el ser humano. La genómica comparada estudia las semejanzas y diferencias entre genomas de diferentes organismos; trata de explicar la información proporcionada por la selección natural para entender la función y los procesos evolutivos que actúan sobre los genomas. La complejidad de los procesos evolutivos constituye todo un desafío a la hora de analizar e interpretar la información biológica generada; en este sentido, la Bioinformática y la Biología Computacional proporcionan un amplio abanico de técnicas estadísticas, matemáticas y algorítmicas para el análisis de datos biológicos. Un reto clave es analizar el caudal de datos de secuencias de ADN con el fin de comprender la información almacenada en términos de estructura, función y evolución proteicas. En Biología computacional BLAST y ClustalW2 son las herramientas más empleadas para el análisis de alineamientos múltiples de secuencias. La Genómica está resultando clave también en el campo de la medicina; conocer la cartografía del genoma humano proporciona una valiosa información a tener en cuenta a la hora de detectar genes implicados en ciertas enfermedades. Esto conlleva que en la actualidad nos centremos más en la predicción de patologías que en la prevención, por lo que la tendencia es que en el futuro la Medicina Genómica acabe desbancando a la Medicina Preventiva. El Proyecto Genoma Humano presenta diversas aplicaciones que, al no tener una clara cobertura legal, traen consigo un nuevo paradigma con problemas éticos, sociales y legales que la comunidad científica trata de resolver para compaginar los aspectos morales con el progreso en la investigación.
\end{abstract}

PALABRAS CLAVE: Cordados; Genoma; Genómica Comparativa; Medicina Genómica; Ética.

\section{INTRODUCCIÓN}

Todos los seres vivos se agrupan dentro de 35 filas que abarcan la inmensa diversidad morfológica generada durante el proceso de evolución animal. Cada una de estas morfologías ha derivado de un mecanismo de desarrollo embrionario regulado por infinidad de genes que interactúan entre sí. El proceso evolutivo conlleva la generación de nuevas especies, a partir de otras preexistentes o ancestros, que comparten determinadas características morfológicas con la especie de la que derivan.

Dentro del Reino Animal se halla el filo de los Cordados, individuos cuya principal característica es una cuerda dorsal (notocorda) presente durante el desarrollo o alguna de sus fases. Son animales de morfología muy variada, adaptados a ambientes ecológicos muy diversos y que a lo largo de su proceso evolutivo han desarrollado una serie de cualidades que les han permitido situarse como eslabones tróficos finales de sus respectivos ecosistemas. En ellos destaca su capacidad de autorregulación y organización interna, lo que les confiere cierta complejidad, y les permite controlar mejor sus reacciones metabólicas y desarrollar un complejo sistema nervioso. Actualmente se conocen unas 65.000 especies pertenecientes a este filo, entre las que se incluye el ser humano, que concretamente pertenece al subfilo vertebrados (Gállego Castejón, 2006).

El origen de los Cordados es una cuestión que genera discusión desde mediados del pasado siglo XX, cuando la teoría de la evolución resultó primordial para la búsqueda de relaciones entre los diferentes grupos de organismos. El espectacular desarrollo de la Biología Molecular durante la última mitad del siglo XX nos ha permitido conocer la natu-

* II Biología Celular, Histología y Embriología, Informática Médica, Facultad de Ciencias Médicas. Universidad Nacional de Córdoba, Argentina.

**Unidad de Biología de Sistemas y Organismos, Dpto. Biología Experimental, Universidad de Jaén, España. 
raleza del material genético, descifrar su código y desentrañar los mecanismos básicos de control de la expresión génica, sentando las bases para el desarrollo de la biotecnología.

Cada individuo tiene la información genética almacenada en el ADN de todas sus células, la cual guarda gran similitud $(99,8 \%)$ con todos los de su propia especie. Esa información que lo define e identifica como ser único e independiente, es lo que conocemos como patrimonio genético o genoma (Samar et al., 2006).

El conocimiento de la secuencia del genoma humano supuso un avance sin precedentes en el campo de la genética y ha sido el acontecimiento científico de mayor relevancia ocurrido en los albores del siglo XXI. La secuencia del genoma humano contiene la información genética almacenada en cada una de las células de una persona. La secuenciación del genoma no sólo nos devela la clave genética necesaria para la creación de un ser humano, sino que además arroja luz sobre aspectos relacionados con nuestros orígenes y nos aporta nuevas perspectivas para combatir enfermedades (Salas, 2004).

En el último cuarto del siglo XX y en los primeros años de este siglo XXI se ha secuenciado de forma completa el genoma de infinidad de organismos; dicha secuencia puede considerarse el "mapa genético supremo", en el sentido de que toda la información hereditaria se encuentra codificada dentro del ADN y el orden en que se sitúan los nucleótidos a lo largo de cada cromosoma es perfectamente conocido. Sin embargo, el conocimiento de la secuencia de ADN de un organismo no nos devela cómo la información contenida en el ADN da lugar a un determinado fenotipo. Encontrar las partes funcionales de las secuencias genómicas y utilizar esta información en biomedicina y genética clínica es el principal objetivo a cumplir. El análisis comparativo de secuencias genómicas desempeña una función primordial para ello.

La mayor parte de la dotación genética humana proviene de un pasado lejano desde el punto de vista evolutivo. Las características comunes de dos organismos permanecerán codificadas dentro del $\mathrm{ADN}$ que se conserva entre especies. Es decir, las funciones celulares básicas, como el metabolismo, el proceso de transcripción del ADN en ARN, la traducción del ARN en proteínas, o la replicación del ADN, evolucionaron una única vez y han permanecido prácticamente inalterables desde la evolución de los organismos unicelulares.

Por tanto, ¿qué distingue un organismo de otro? Cabe esperar que secuencias que codifiquen o controlen la expresión de proteínas y ARNs responsables de diferencias entre especies serán divergentes. Así, existen datos que corroboran la aparición en los vertebrados de dos tipos de genes: los específicos de cualidades características como la complejidad neuronal, la coagulación de la sangre o la inmunidad adquirida, y aquellos que otorgan cualidades más generales, como genes para el proceso de transducción de señales intra e intercelulares, el desarrollo, la apoptosis o el control de la expresión génica. Conocer la secuencia de los genomas de otros organismos es pues fundamental a la hora de intentar discernir cómo se ha llevado a cabo el proceso evolutivo (Cañestro et al., 2007).

El objetivo del presente trabajo es describir brevemente conceptos de la genómica de los cordados y de la genómica comparativa y sus aspectos éticos.

\section{La Genómica Comparativa}

La genómica comparada se ocupa de las similitudes y discrepancias existentes entre genomas de distintos organismos. Una de sus principales aplicaciones es la predicción de genes así como el descubrimiento de nuevos y no codificantes, pero funcionales, elementos del genoma. La genómica comparada se sirve de las semejanzas y divergencias existentes en las proteínas, el ARN y las regiones reguladoras de distintos organismos para intentar determinar cómo el proceso de selección natural ha intervenido sobre tales elementos. Se mantendrían estables en el tiempo aquellos elementos que confieran semejanzas entre distintas especies (selección estabilizadora), mientras que los elementos causantes de las discrepancias entre especies deberían divergir (selección direccional). Por último, aquellos elementos que no aportan nada relevante desde el punto de vista del proceso evolutivo del organismo no serán conservados (selección neutral) (Hardison, 2003).

Uno de los objetivos primordiales de la genómica comparada es la identificación de los mecanismos evolutivos del genoma eucariota. No obstante, esta tarea es bastante ardua dada la multiplicidad de eventos transcurridos a través de la historia de linajes individuales, eventos que a su paso sólo han dejado trazas distorsionadas y superpuestas en el genoma de cada organismo vivo. Por esta razón, los estudios por genómica comparada de pequeños organismos modelo son de gran importancia para avanzar en nuestro conocimiento de los mecanismos generales de la evolución.

La genómica comparada ha jugado también un importante papel en el estudio de la evolución de los cordados. Se cree que los cefalocordados, urocordados y vertebrados evolucionaron a partir de un ancestro común hace más de 520 millones de años. En un intento de comprender la evolución de este filo y el origen de los vertebrados, diversos 
estudios (Holland et al., 2008; Putnam et al., 2008) se han centrado en buscar intensivamente genes concretos, familias de genes y elementos no codificantes altamente conservados en el genoma secuenciado del cefalocordado Branchiostoma floridae, llamado vulgarmente anfioxo.

Se ha prestado especial atención a los genes Hox, específicos del desarrollo y diferenciación de las diversas regiones del cuerpo y cuya función es universal para todo el Reino Animal, incluyendo la especie humana, a los genes implicados en el desarrollo de la cresta neural y a los genes que codifican componentes de los sistemas inmune y endocrino, entre otros. Así, se ha podido comprobar que el genoma del anfioxo contiene un set básico de genes de cordados implicados en procesos de desarrollo y señalización celular (Osbornen \& Ferrier, 2010)

\section{El genoma del anfioxo como antecedente para entender la evolución genética de los vertebrados.}

El estudio de genes clave en el desarrollo del anfioxo ha arrojado luz sobre la evolución de órganos de vertebrados tales como el cerebro, los riñones, el páncreas y la hipófisis, así como sobre los mecanismos genéticos de los patrones embrionarios primarios en general. Asimismo, la secuencia genómica del anfioxo comparada con los genomas de otros organismos, revela características clave del genoma del último ancestro común de todos los cordados y ha permitido esclarecer su árbol filogenético (Putnam et al.).

De este estudio evolutivo se desprende que este antecesor común vivió probablemente antes del período Cámbrico y a partir de él surgiría el linaje de los cordados, actualmente representado por los cefalocordados modernos como el anfioxo, los urocordados y los vertebrados. De los linajes que actualmente siguen vivos, los cefalocordados divergerían primero, antes de la separación entre los morfológicamente diversos urocordados y vertebrados.

Es más, el genoma del anfioxo se perfila como un buen representante del ancestral genoma de los cordados en relación al contenido en genes, la estructura de exonesintrones e incluso la organización cromosómica. Cabe destacar también que pequeños niveles de secuencias no codificantes han sido conservadas entre anfioxo y humano (siendo las regiones no codificantes más antiguas detectadas a través de alineación de secuencia directa) proporcionando una visión tentadora del sistema de regulación génica del último ancestro común del filo cordados.

Aunque inicialmente la genómica comparada se empleaba para la identificación de proteínas funcionales, los esfuerzos están ahora concentrados en descubrir regiones reguladoras y moléculas de ARNip (ARN interferente pequeño, del inglés small interfering RNA o siRNA). Hace relativamente poco tiempo que se ha descubierto que especies lejanamente relacionadas comparten a menudo largos tramos de ADN conservados que aparentemente no codifican ninguna proteína. Aunque por ahora se desconoce la funcionalidad de tales regiones ultraconservadas, predecir con exactitud cuál sería la función de dichas secuencias no codificantes constituye todo un desafío (Richard et al., 2008).

Conforme la ciencia va avanzando hacia el entendimiento biológico a niveles sistémicos, el acceso a grandes cantidades de datos de muy diferente naturaleza se ha convertido en algo crucial. Tecnologías tales como la secuenciación de genomas, la genómica comparada, los microarrays, la proteómica y la genómica estructural nos han proporcionado listas parciales y totales para muchos organismos vivos. La predicción de genes, de la estructura de proteínas y de la expresión génica, el alineamiento de secuencias y el ensamblaje del genoma, las interacciones entre proteínas y el modelado de la evolución se perfilan como las principales líneas de investigación a tener en cuenta.

Los investigadores se centran ahora en cómo los componentes individuales encajan juntos para construir los sistemas biológicos. Sin embargo, esta revolución biotecnológica también amenaza con sepultarnos bajo grandes cantidades de datos que es necesario interpretar y analizar de manera correcta; es por ello que actualmente hay una creciente necesidad de recoger, almacenar y depurar toda esta información biológica de tal manera que nos permita su eficiente recuperación y explotación.

\section{Análisis de genomas. Bases de datos de Genómica Com- parativa.}

Para el análisis y la gestión de toda esta gran cantidad de información biológica se requiere de la bioinformática y la biología computacional. Estas herramientas nos permiten el análisis de datos y la simulación de sistemas o mecanismos biológicos mediante el empleo de algoritmos matemáticos, aproximaciones y programas bioinformáticos.

En el caso concreto de la Genómica Comparada, el análisis se centra en establecer correspondencias entre genes u otras características genómicas de distintos organismos. Los mapas intergenómicos que obtenemos como resultado de estos análisis ortólogos son los que permiten el rastreo de los procesos evolutivos que ocasionan las divergencias genómicas. La compleja evolución del genoma plantea muchos desafíos a la hora de desarrollar modelos matemáticos $\mathrm{y}$ algoritmos que puedan explicarla, lo que hace necesario recurrir a un amplio abanico de técnicas algorítmicas, esta- 
dísticas y matemáticas. La gran mayoría de estos estudios se basan en detectar homologías y computar familias de proteínas.

La bioinformática emplea como herramientas de software desde simples líneas de comandos hasta programas gráficos de una mayor complejidad (Goujon et al., 2010).

Los recursos informáticos públicos disponibles en Internet (basados en la web y disponibles para los tres sistemas operativos, Win, Mac, Linux) son la mayoría gratuitos y ha tenido un tremendo impacto sobre la capacidad de los científicos para presentar y diseminar los resultados de sus investigaciones. Las principales bases de datos de ácidos nucleicos recogen una fracción de los datos totales de secuencias producidas en todo el mundo y que intercambian los registros nuevos y los actualizados diariamente (Nielsen et al., 2010).

En Biología computacional BLAST y ClustalW2 son las herramientas más empleadas para el análisis de alineamientos múltiples de secuencias. El primero es sin duda el más conocido, se trata de un algoritmo que nos permite determinar similitudes entre secuencias arbitrarias y secuencias almacenadas en bases de datos de secuencias de ADN o proteínas. Una implementación ampliamente utilizada, que se basa en web y trabaja sobre sus propias bases de datos la podemos encontrar en el NCBI (National Center for Biotechnology Information, USA).

ClustalW2 es también un software de referencia; el EBI (European Bioinformatics Institute) ha realizado una implementación del mismo que está siendo de gran utilidad.

Aparte de los mencionados anteriormente, gran cantidad de software o programas bioinformáticos ha surgido con otros objetivos como el alineamiento estructural de proteínas, la predicción de genes, predicción de estructura de proteínas, predicción de acoplamiento proteínaproteína, o el modelado de sistemas biológicos (Nielsen et al.).

La creación de interfaces para una gran variedad de aplicaciones bioinformáticas ha supuesto una gran ventaja para los usuarios, no sólo porque éstas permiten ejecutar una aplicación en un ordenador cualquiera empleando algoritmos, datos y otros recursos computacionales alojados en servidores localizados en cualquier otro punto del planeta, sino porque además, el usuario final puede despreocuparse de actualizaciones y modificaciones en el software o en las bases de datos.
Hoy en día, conforme el número de genomas secuenciados va en aumento, los grandes estudios de genómica comparada se están volviendo extremadamente costosos desde el punto de vista computacional. Es por ello que la Computación en Nube (Cloud computing) está emergiendo como una solución robusta y económica para llevar a cabo procesos computacionales de todo tipo. Aunque hasta la fecha esta técnica de análisis ha tenido un impacto limitado en genómica comparada, recientes estudios (Wall et al., 2010) han demostrado que se perfila como una buena alternativa para efectuar análisis computacionales de alto rendimiento.

\section{Medicina Genómica. Aspectos éticos, legales y sociales del Genoma Humano.}

Si bien los genes no tienen como función causar enfermedades, fueron los defectos genéticos asociados a distintas enfermedades los que principalmente sirvieron de marcadores para catalogar los genes correspondientes (Dosne Pasqualini, 2001). Las enfermedades genéticas son reconocidos como una de las principales en las categorías de enfermedades humanas (Kumar, 2008).

La medicina genómica es el uso de la información de los genomas y sus derivados (ARN, proteínas y metabolitos) y permite guiar la toma de decisiones médicas, es un componente clave de la medicina personalizada (Ginsburg \& Willard, 2009; Feero et al., 2010).

Así pués, lo adelantos tecnológicos en relación al conocimiento del genoma humano han llevado al surgimiento de la terapia génica. La terapia génica se basa en la aplicación de la tecnología del ADN recombinante (Ingeniería Genética) para modificar el gen defectuoso o reemplazarlo por el gen normal, de manera permanente, o bien silenciar genes (Capó \& Frejo, 2006).

La medicina comienza a adoptar las herramientas genómicas que permitan la predicción más precisa y tratamiento de enfermedades. Las tecnologías de secuenciación de ADN y los análisis de transcriptomas, proteomas y metabolomes han proporcionado las bases para descifrar la estructura, la variación y la función del genoma humano y de relacionarlos con la salud y la enfermedad. (Wall et al., 2009; Thompson et al., 2010).

La mayor eficacia de la secuenciación de ADN se abre la posibilidad de analizar un gran número de genomas individuales y transcriptomas, y proteomas completos de referencia y metabolomes están dentro de alcance utilizando potentes técnicas de análisis basadas en cromatografía, espectrometría de masas y resonancia magnética nuclear. 
Actualmente se propone la medicina sistémica como futuro de la medicina genómica. Esta especialidad permitirá a la medicina convertirse en predictiva, personalizada, preventiva y participativa (Auffray et al., 2009).

El Genoma Humano trajo aparejado un nuevo paradigma con problemas éticos, sociales y legales (Dosne Pasqualini, Samar \& Avila, 2002; Samar et al.; Ávila et al., 2008).

Luego de un amplio debate que demandó la redacción de ocho anteproyectos en un periodo de cuatro años, el 25 de junio de 1997 el texto final de la declaración fue aprobado por la Comisión de expertos de UNESCO. La Declaración fue aprobado por unanimidad y por aclamación por la Conferencia General en su reunión No 29 del 11 de noviembre de 1997, primer instrumento universal en el campo de la Biología. El mérito indiscutible de ese texto radica en el equilibrio que establece entre la garantía del respeto de los derechos y las libertades fundamentales, y la necesidad de garantizar la libertad de la investigación.

Los grandes temas de la Declaración son:

a. La dignidad humana y el genoma humano. Donde se considera en sentido simbólico que el genoma humano es patrimonio de la humanidad.

b. Derechos de las personas interesadas. Donde se revalorizan el consentimiento informado, la confidencialidad y la no discriminación por cuestiones genéticas.

c. Investigaciones sobre el genoma humano. Donde se regulan las investigaciones científicas supeditando la libertad de pensamiento y de investigación a los derechos de los individuos en relación a su dignidad y libertad.

d. Condiciones de ejercicio de la actividad científica. Donde se establecen los derechos y responsabilidades de los investigadores.

e. Solidaridad y cooperación internacional. Donde se abordan los principios de solidaridad y cooperación internacional tanto con las personas como con los pueblos y comunidades vulnerables.

Es de destacar que esta Declaración no avanza en la controversia en relación al comienzo de la vida, tema sobre el cual la Comisión no logró consenso. Así, ciertos autores consideran que la persona existe desde el momento de la concepción, correspondiente a la formación del cigoto. Sostienen que en el momento de la fecundación los dos gametos forman una nueva entidad biológica, el cigoto o embrión celular, una nueva vida individual, una persona con un código genético individualizado, un genoma que individualiza y define al hombre, diferente al de sus progenitores. Desde ese momento de la concepción se produce el desarrollo biológico ininterrumpido de ese nuevo individuo humano, cumpliendo un ciclo o curva vital. Mantienen que cada ser humano es un ser único e irrepetible en la historia de la humanidad (Ávila et al.). Otros autores, sin embargo, defienden un punto de vista diferente, argumentando que la falta de un desarrollo fisiológico adecuado y necesario para el mantenimiento de las capacidades sensitivas hace que los embriones no sean candidatos apropiados a adscribirse a los derechos humanos y morales que se les quiere atribuir (Warren, 1991).

Un principio ético fundamental en relación al conocimiento del genoma humano es el de proteger la intimidad de los datos genéticos personales, evitando su difusión a terceras personas o entidades (Williams \& Schroeder, 2004; Petersen et al., 2005; Fan et al., 2008). En todo caso, la inviolabilidad del genoma humano debe también considerarse como un principio a mantener de forma clara y absoluta; desde el punto de vista ético no se puede alterar el genoma propio de la especie humana. Actualmente los esfuerzos se centran en dilucidar el camino a seguir en un futuro inmediato para garantizar la ética sin llegar a cortar el cauce de conocimientos y progresos aportados por el espectacular desarrollo de la Genética Molecular.

ÁVILA, R. E.; SAMAR, M. E.; DÍAZ-BELTRÁN, L. \& ESTEBAN, F. J. The genome in the chordates: introduction to comparative genomics. Int. J. Morphol., 30(4):1309-1315, 2012.

SUMMARY: The aim of this paper is to briefly describe concepts of genomics of chordates and comparative genomics and its ethical aspects. The genome of chordates changed slightly resulting in the genome of vertebrates, including mammals. The human being belongs to the phylum chordates. Comparative genomics studies the similarities and differences between genomes of different organisms, trying to explain the information provided by natural selection to understand the function and evolutionary processes that act on genomes. Evolutive processes' complexity is considered a major challenge in terms of analyzing and interpreting all the biological information generated; in this sense, Bioinformatics and Computational Biology provide an enormous range of statistical, mathematical and algorythmical techniques for biological data analyses. A key challenge for bioinformatics is to analyze the flow of DNA sequence data to understand the information stored in terms of structure, function and protein evolution. BLAST and ClustalW2 tools are used for the analysis of multiple 
sequence alignments in Computational Biology. Genomics is also playing a key role in Medicine; human genome's cartography provides valuable information to detect genes involved in certain diseases. It entails that nowadays it is better to focus on prediction much more than on prevention. The current tendency is that in the future Genomic Medicine will displace Preventive Medicine. The Human Genome Project implies diverse applications that do not have clear legal coverage. This brings a new paradigm with ethical, social and legal problems that the scientific community is trying to resolve in order to combine morality and research progress.

KEY WORDS: Chordates; Genome; Comparative genomic; Genomic medicine; Ethic.

\section{REFERENCIAS BIBLIOGRÁFICAS}

Auffray, C.; Chen, Z. \& Hood, L. Systems medicine: the future of medical genomics and healthcare. Genome Med., 1(1):2, 2009.

Ávila, R. E.; Samar, M. E. \& Ferraris, R. V. El embrión como persona y paciente. Córdoba. Argentina, Editorial SeisC, 2008.

Capó, M. A. \& Frejo, M. T. Toxicogenómica, una nueva rama de la toxicología. Medicina Balear [en línea] 22(3), 2007. Disponible en: http://dialnet.unirioja.es/servlet/ articulo?codigo $=2555755$.

Cañestro, C.; Yokoi, H. \& Postlethwait, J. H. Evolutionary developmental biology and genomics. Nature reviews genetics, 8:932-42, 2007.

Dosne Pasqualini, C. El Genoma Humano. Medicina (B. Aires), 61:243-6, 2001. Disponible en: http:// www.medicinabuenosaires.com/revistas/vol61-01/2/ genomahumano.htm

Fan, C. T.; Wu, C. \& Shi, Z. Impact of development of population-based study in biomedical field on laws and regulations: a cross-strait experience on biobank development. J. Int. Bioethique., 19(4):117-37, 166, 2008.

Feero, W. G.; Guttmacher, A. E. \& Collins, F. S. Genomic medicine--an updated primer. N. Engl. J. Med., 362(21):200111,2010 .

Gállego Castejón, L, Los Cordados: origen y diversificación. San Vicente (Alicante), Editorial Club Universitario, 2006.

Ginsburg, G. S. \& Willard, H. F. Genomic and personalized medicine: foundations and applications. Transl. Res., 154(6):277-87. 2009.
Goujon, M.; McWilliam, H.; Li, W.; Valentin, F.; Squizzato, S.; Paern, J. \& Lopez, R. A new bioinformatics analysis tools framework at EMBL-EBI. Nucleic Acids Res., 38Suppl:W695-9., 2010.

Hardison, R. C. Comparative Genomics. PLoS Biology, 1(2):E58, 2003.

Holland, L. Z.; Albalat, R.; Azumi, K.; Benito-Gutiérrez, E.; Blow, M. J.; Bronner-Fraser, M.; Brunet, F.; Butts, T.; Candiani, S.; Dishaw, L. J.; Ferrier, D. E.; Garcia-Fernàndez, J.; Gibson-Brown, J. J.; Gissi, C.; Godzik, A.; Hallböök, F.; Hirose, D.; Hosomichi, K.; Ikuta, T.; Inoko, H.; Kasahara, M.; Kasamatsu, J.; Kawashima, T.; Kimura, A.; Kobayashi, M.; Kozmik, Z.; Kubokawa, K.; Laudet, V.; Litman, G. W.; McHardy, A. C.; Meulemans, D.; Nonaka, M.; Olinski, R. P.; Pancer, Z.; Pennacchio, L. A.; Pestarino, M.; Rast, J. P.; Rigoutsos, I.; Robinson-Rechavi, M.; Roch, G.; Saiga, H.; Sasakura, Y.; Satake, M.; Satou, Y.; Schubert, M.; Sherwood, N.; Shiina, T.; Takatori, N.; Tello, J.; Vopalensky, P.; Wada, S.; Xu, A.; Ye, Y.; Yoshida, K.; Yoshizaki, F.; Yu, J. K.; Zhang, Q.; Zmasek, C. M.; de Jong, P. J.; Osoegawa, K.; Putnam, N. H.; Rokhsar, D. S.; Satoh, N. \& Holland, P. W. The amphioxus genome illuminates vertebrate origins and cephalochordate biology. Genome Res., 18(7):1100-11, 2008.

Kumar, D. Disorders of the genome architecture: a review. Genomic Med., 2:69-76, 2008.

Nielsen, C. B.; Cantor, M.; Dubchak, I.; Gordon, D. \& Wang. T. Visualizing genomes: techniques and challenges. Nature Methods, 7:S5-S15, 2010.

Osborne, P. W. \& Ferrier, D. E. Chordate Hox and ParaHox gene clusters differ dramatically in their repetitive element content. Mol. Biol. Evol., 27(2):217-20, 2010.

Petersen A. Securing our genetic health: engendering trust in UK Biobank. Sociol. Health Illn., 27(2):271-92, 2005.

Putnam, N. H.; Butts, T.; Ferrier, D. E.; Furlong, R. F.; Hellsten, U.; Kawashima, T.; Robinson-Rechavi, M.; Shoguchi, E.; Terry, A.; Yu, J. K.; Benito-Gutiérrez, E. L.; Dubchak, I.; Garcia-Fernàndez, J.; Gibson-Brown, J. J.; Grigoriev, I. V.; Horton, A. C.; de Jong, P. J.; Jurka, J.; Kapitonov, V. V.; Kohara, Y.; Kuroki, Y.; Lindquist, E.; Lucas, S.; Osoegawa, K.; Pennacchio, L. A., Salamov, A. A.; Satou, Y.; SaukaSpengler, T.; Schmutz, J.; Shin-I, T., Toyoda, A.; BronnerFraser, M.; Fujiyama, A.; Holland, L. Z.; Holland, P. W.; Satoh, N. \& Rokhsar, D. S. The amphioxus genome and the evolution of the chordate karyotype. Nature, 453(7198):1064-71, 2008.

Richard, G. F.; Kerrest, A. \& Dujon, B. Comparative genomics and molecular dynamics of DNA repeats in eukaryotes. Microbiol. Mol. Biol. Rev., 72(4):686-727,2008. 
ÁVILA, R. E.; SAMAR, M. E.; DÍAZ-BELTRÁN, L. \& ESTEBAN, F. J. El genoma en los cordados: introducción a la genómica comparada. Int. J. Morphol., 30(4):1309-1315, 2012.

Salas, M. Repercusiones médicas del descubrimiento del genoma humano. Ochoa y la medicina clínica. Farmaindustria, Serie Cientìfica, Madrid, Capítulo 4.57-70, 2004. Disponible en: http://www.ujaen.es/investiga/ inmunoge/gmo/articulos.htm

Samar, M. E. \& Avila R. E. Problemática jurídico-legal de la fertilización asistida: la persona y el comienzo de la vida. Rev. Claves Odontol., 49:7-9, 2002.

Samar, M. E.; Arriaga, A. \& Avila, R. E. El genoma humano. Manual de Medicina. Editorial Antinori, Córdoba, 2006.

Thompson, P. M.; Martin, N. G. \& Wright, M. J. Imaging genomics. Curr. Opin. Neurol. 23(4):368-73, 2010.

Wall, D. P.; Esteban, F. J.; Deluca, T. F.; Huyck, M.; Monaghan, T.; Velez de Mendizabal, N.; Goñí, J. \& Kohane, I. S. Comparative analysis of neurological disorders focuses genome-wide search for autism genes. Genomics, 93(2):1209, 2009.

Wall, D. P.; Kudtarkar, P.; Fusaro, V. A.; Pivovarov, R.; Patil, P. \& Tonellato, P. J. Cloud computing for comparative genomics. BMC Bioinformatics, 11:259, 2010.

Warren, M. A. Embryo manipulation and experimentation. Baillieres Clin. Obstet. Gynaecol., 5(3):591-609, 1991.

Williams, G. \& Schroeder, D. Human genetic banking: altruism, benefit and consent. New Genet. Soc., 23(1):89-103, 2004.

\section{Dirección para correspondencia: \\ Prof. Dr. Rodolfo E. Ávila \\ Calle Catamarca 1546 (5000) \\ Córdoba, \\ ARGENTINA}

E-mail: avilainfo@gmail.com

Recibido : 18-02-2012

Aceptado: 27-05-2012 